\title{
Barriers to Acceptance of Provider-Initiated Testing and Counseling among Men Who Have Sex with Men in Shenyang, China: A Cross-Sectional Study
}

\author{
Qinghai Hu, Junjie Xu, Zhenxing Chu, Jing Zhang, Ke Yun, Feng Shi, \\ Yongjun Jiang, Wenqing Geng, and Hong Shang
}

Key Laboratory of AIDS Immunology of the Ministry of Health, Department of Laboratory Medicine, No. 1 Hospital of China Medical University, 155 Nanjing North Street, Shenyang 110001, China

Correspondence should be addressed to Hong Shang; hongshang100@hotmail.com

Received 8 April 2013; Accepted 17 June 2013

Academic Editor: Lucia Lopalco

Copyright (C) 2013 Qinghai Hu et al. This is an open access article distributed under the Creative Commons Attribution License, which permits unrestricted use, distribution, and reproduction in any medium, provided the original work is properly cited.

\begin{abstract}
A high prevalence of HIV infection is present among men who have sex with men (MSM) in China, but many people living with HIV or AIDS (PLWHs) are unaware of their HIV infection status. Provider-initiated HIV testing and counseling (PITC) is a streamlined model that can significantly enhance HIV detection and detect infections earlier. However, PITC has not yet been widely applied, and no studies have been conducted on MSM's attitudes towards PITC in China. In this study, a total of 438 MSM were recruited in Shenyang city. A multivariate logistic regression model showed that certain conditions made MSM more accepting of PITC: those who had attended VCT (voluntary counseling and testing) more than three times (odds ratio [OR]: 2.95, 95\% CI: 1.36-6.37), those who considered PITC beneficial for family and friends (OR: 1.91, 95\% CI: 1.25-2.92), those who obtained HIV/AIDS knowledge from brochures (OR: 2.52, 95\% CI: 1.64-3.87), those who obtained HIV/AIDS knowledge from the Internet (OR: 1.66, 95\% CI: 1.07-2.58), and those who were highly aware of their own risk of being infected with HIV (OR: 2.84, 95\% CI: 1.37-5.91). To improve acceptance of PITC among MSM in China, stronger efforts are needed to lower the psychosocial barriers to receiving PITC, to promote HIV/AIDS awareness, and to encourage the extension of HIV testing.
\end{abstract}

\section{Introduction}

Human immunodeficiency virus (HIV) testing is the first step in many HIV prevention, care, and treatment programs because testing allows individuals who are at high risk for HIV infection to learn about their infection status and access appropriate services $[1,2]$. As antiretroviral therapy (ART) becomes more widely available in resource-limited settings, increasingly, the challenge is case identification so that patients may benefit from ART in a timely fashion and improve their quality of life. Moreover, HIV testing may also help to prevent HIV secondary transmission [3]. Data from across China show there are an estimated 780,000 people living with HIV/AIDS (PLWHs) in 2011, and, unfortunately, more than $56 \%$ of PLWHs are unaware they have been infected [4]. Identifying these people and the status of their infection has never been more important and urgent.
There are currently an estimated 18 million men engaging in homosexual sex in China [5]. More importantly, HIV transmission among homosexuals rose from $0.3 \%$ before 2005 to more than $13.7 \%$ in $2011[4,5]$. Thus, in addressing the China AIDS epidemic, it is critical to control the spread of HIV among MSM.

Voluntary counseling and testing (VCT) for HIV has been demonstrated to be one of the most effective strategies in identifying HIV-infected individuals $[1,6]$. VCT was highly efficacious in reducing risky sexual risk behaviour, especially for high-risk populations [1, 7]. However, there are also barriers associated with VCT participation, which include clients having to initiate testing themselves, lengthy pre- and posttest counseling, and patient concerns about confidentiality $[8,9]$. Because of the double discrimination they face because of their sexual orientation and being HIVinfected, Chinese MSM have great fear of being exposed. 
Thus, most Chinese MSM experience high affective barriers to proactively take part in VCT for HIV.

Provider-initiated HIV testing and counseling (PITC) is a streamlined model promoted by the World Health Organization (WHO) in 2007 [10]. WHO policy guidelines suggest that HIV testing and counseling has to be offered by health care providers as part of the routine care of all patients or clients [10-12]. Compared with VCT, PITC simplifies information acquisition and counseling about high-risk behaviour and allows MSM to avoid the "humiliation" and "inconvenience" they fear. In this way, PITC can increase HIV detection rates, catch HIV infections earlier, and, thus, improve cost-effectiveness by cutting down on treatment costs $[10,13,14]$. A study from The Netherlands found that a PITC strategy decreased the rates of MSM refusals to receive HIV testing from $38 \%$ in 2006 to $12 \%$ in 2007 [15]. Also because of PITC, the rate of HIV detection by hospitals increased from $10.7 \%$ in 2005 to $29.4 \%$ in 2008 in Yunnan Province (Lin Lu, unpublished data). Nearly $71.4 \%$ of those who were found to be HIV positive in the clinic of the First Affiliated Hospital of China Medical University selfidentified as MSM (Hong Shang, unpublished data), which suggests that the PITC carried out in the hospital seemed to be especially beneficial among MSM and, possibly, other high-risk groups. Some studies from the USA and Uganda have shown that PITC has been adopted by between $53 \%$ and $95 \%$ of the emergency departments in these countries and has led to substantial increases in participation in HIV testing $[16,17]$. However, the psychosocial factors associated with PITC acceptance have not been previously examined among Chinese MSM.

The primary aim of this study was to evaluate the acceptability of PITC among MSM in Shenyang, China. Shenyang is an economic, cultural, and industrial hub of Liaoning province. Shenyang has the second largest MSM population in China (after Beijing) with an estimated 140,000 MSM who are from the city [18]. Previous regional studies of Shenyang and its surrounding areas revealed both high HIV seroincidence [19] and high prevalence among local MSM [20], but there have been no studies examining MSM attitudes towards PITC. Exploring the willingness of Shenyang MSM to accept PITC will help inform intervention policies or strategies for MSM throughout China.

\section{Materials and Methods}

2.1. Study Design and Participant Enrolment. The study was conducted from June to September 2010, during which MSM were recruited through snowball sampling via a nongovernmental organization (NGO), the Shenyang Sunny Workgroup, which provides health education outreach to Shenyang MSM. Recruited individuals were interviewed at the HIV Voluntary Counseling and Testing Clinic at the First Affiliated Hospital of China Medical University. The inclusion criteria for participating in the study were male, HIV negative, at least 18 years of age, reported having had at least one male sexual partner with whom he had receptive and/or insertive anal sex within the past 12 months, and physically able and willing to provide written informed consent.
2.2. Ethics. Ethical approval was obtained from the Institutional Review Board of the First Affiliated Hospital of China Medical University prior to the commencement of the study. Permission to conduct the study was sought from the local administrative authority. Informed consent was obtained from all participants after they received an explanation of the study's aim. For confidentiality and privacy, unique identification numbers were used instead of names.

2.3. Data Collection. An anonymous questionnaire was designed with the primary aim of obtaining information about the subject's acceptance of PITC. Other demographic, cognitive, and behavioral variables were considered to ascertain their influence on the participant's willingness to accept PITC. The majority of the questions have been widely used or reported in other literature. The questionnaire consisted of 31 questions divided into three sections: (1) sociodemographic characteristics, (2) knowledge and attitudes concerning HIV/AIDS and PITC, and (3) sexual behavior. In the first section, participants were asked about basic demographic information, such as nationality, age, ethnicity, education level, job, monthly income, and preferred medium for seeking sexual partners. The second section was designed to determine the subject's willingness to accept PITC as well as knowledge regarding HIV preventionsuch as transmission routes, prevention methods, and correct use of condoms - and their knowledge about the benefits of PITC. In section three, the questions dealt with sexual health and behavior. Interviews were administered face-to-face by trained physicians or volunteers from NGOs. Investigators introduced PITC to all participants before the interview. A small incentive equivalent to USD \$5 was given to each participant as compensation for their time.

2.4. Data Analysis. Questionnaire data were entered twice and then checked for accuracy using Epi Data software (the Epi Data Association Odense, Denmark, version 3.02). Data were then analyzed using SAS 9.1 (SAS Institute Inc., Cary, NC, USA). Descriptive statistics and univariate analysis were generated for each of the variables corresponding to specific questions in the survey.

There were eleven questions related to HIV/AIDS prevention knowledge that participants were asked. Answering one question correctly earned them one point. Bivariate logistic analysis was performed to study the association of each of the independent variable with an expression of interest in PITC (which was considered acceptance of PITC). The majority of these independent variables were dichotomized based on the presence (a "Yes" response) or absence (a "No" response) of the specific characteristic being examined. Age was arbitrarily dichotomized as "older" and "younger" using the approximate median age (28 years) of participants as the cutoff, and high school-level education and 1000 Chinese Yuan (CNY) per month were used as cutoffs for the dichotomous variables "education" and "monthly income", respectively. Contingency tables were constructed for all comparisons and the chisquare $\left(\chi^{2}\right)$ test; Mantel-Haenszel statistics and measures of association were calculated (Odd Ratios). Test statistics were considered significant if $P$ values were less than 0.05 , and $95 \%$ 
confidence intervals were calculated for the measures of association.

Backwards stepwise multivariate logistic regression analysis was performed to identify factors associated with the acceptance of PITC. Variables that were significant in univariate analysis $(P<0.20)$ were assessed for multicollinearity by using variance inflation factors and tolerance. Then, variables without multicollinearity were considered in multivariate analysis. Only variables with $P<0.05$ were kept in the final multivariate logistic model in a stepwise manner.

\section{Results}

3.1. Demographic Characteristics of MSM Participants. A total of 441 MSM were interviewed in the study, but three of them were disqualified from participation because they were less than 18 years of age. Table 1 provides an overall description of the demographic characteristics of the study population. The median age was 28 years with a range between 18 and 75 years of age. The majority of participants were ethnically Han $(84.5 \%)$, and more than half possessed a high schoollevel education or higher $(57.0 \%)$. For $47 \%$ of the participants (205), the Internet was their preferred medium for seeking sexual partners.

3.2. Behavioral Characteristics of MSM Participants. We found that $22.4 \%$ of the participants had engaged in sex with men, and $13.5 \%$ had engaged in sex with women before the age of 18. About 296 participants (67.6\%) had had regular male sexual partners in the past 12 months, and only $24.7 \%(73 / 296)$ used condoms with these partners during every instance of sexual intercourse. In addition, 352 participants $(80.4 \%)$ had casual male sexual partners in the past 12 months, and $28.7 \%$ of them (101/352) used condoms with these sexual partners during every instance of sexual intercourse. About 400 participants (91.3\%) had previously been tested for HIV. The average scores of AIDS prevention knowledge were 8.8 points out of 11 , and about 93 participants (21.2\%) answered all questions correctly. There were 291 participants $(66.4 \%)$ who obtained their knowledge from the Internet. Of all the participants, 266 (60.7\%), 287 (65.5\%), and 279 (63.7\%) obtained their knowledge from brochures, friends, and televised public advertisements, respectively.

3.3. Willingness to Receive PITC. The number of people who identified themselves as being at high risk for HIV infection was 50 (11.4\%); 259 (59.1\%) self-identified as being at low risk for HIV infection; and 97 (22.2\%) self-identified as being at no risk for $\mathrm{HIV}$ infection. After receiving an explanation about PITC, $53.7 \%$ of participants reported that they would be willing to receive PITC if the procedure was offered to them. The major reasons for declining PITC were the fear of violation of privacy/rights and self-identification as being at no risk or low risk for HIV infection.

Table 2 displays the factors that significantly correlate with the willingness to receive PITC. Correlations were drawn using univariate analysis in which certain factors were found to be significantly associated with the
TABLE 1: Sociodemographic and behavioral characteristics of MSM $(n=438)$

\begin{tabular}{|c|c|}
\hline Demographic/behavioral characteristics & $\begin{array}{c}\text { Number of baseline } \\
\text { MSM } \\
\text { (\%, Proportion) }\end{array}$ \\
\hline \multicolumn{2}{|l|}{ Hometown } \\
\hline Liaoning & $322(73.5)$ \\
\hline Others & $116(26.5)$ \\
\hline \multicolumn{2}{|l|}{ Age (years) } \\
\hline Younger $(<28)$ & $232(53.0)$ \\
\hline Older $(\geqslant 28)$ & $206(47.0)$ \\
\hline \multicolumn{2}{|l|}{ Ethnic } \\
\hline Han & $370(84.5)$ \\
\hline Others & $67(15.3)$ \\
\hline \multicolumn{2}{|l|}{ Education } \\
\hline At least high school & $250(57.1)$ \\
\hline Less than high school & $188(42.9)$ \\
\hline \multicolumn{2}{|l|}{ Occupation } \\
\hline Unemployed & $287(65.5)$ \\
\hline Employed & $148(33.8)$ \\
\hline \multicolumn{2}{|l|}{ Monthly income (RMB) } \\
\hline$\geqslant 1000$ & $289(66.0)$ \\
\hline$<1000$ & $149(34.0)$ \\
\hline \multicolumn{2}{|l|}{ Looking for sexual partners place } \\
\hline Internet & $205(46.8)$ \\
\hline Park & $133(30.4)$ \\
\hline Others & $93(21.2)$ \\
\hline \multicolumn{2}{|l|}{$\begin{array}{l}\text { Had male regular sexual partner in past } 12 \\
\text { months }\end{array}$} \\
\hline Yes & $296(67.6)$ \\
\hline No & $142(32.4)$ \\
\hline \multicolumn{2}{|l|}{$\begin{array}{l}\text { Consistent condom use with regular male } \\
\text { sexual partner }\end{array}$} \\
\hline Yes & $73(16.7)$ \\
\hline No answer & $145(33.1)$ \\
\hline No & $220(50.2)$ \\
\hline \multicolumn{2}{|l|}{$\begin{array}{l}\text { Had male casual sexual partner in past } 12 \\
\text { months }\end{array}$} \\
\hline Yes & $352(80.4)$ \\
\hline No & $86(19.6)$ \\
\hline \multicolumn{2}{|l|}{$\begin{array}{l}\text { Consistent condom use with casual male } \\
\text { sexual partner }\end{array}$} \\
\hline Yes & $101(23.1)$ \\
\hline No answer & $88(20.1)$ \\
\hline No & $249(56.8)$ \\
\hline
\end{tabular}

willingness to receive PITC ( $P<0.05)$. These factors were self-identification of high risk for HIV infection; HIV/AIDS prevention knowledge scores; HIV testing frequency through VCT; seeing PITC as beneficial for family and friends; obtaining HIV/AIDS prevention knowledge from the radio; obtaining HIV/AIDS prevention knowledge 
TABLE 2: Willingness for accepting PITC among Shenyang MSM by demographic and sexual behavioral characteristics (unadjusted OR).

\begin{tabular}{|c|c|c|c|c|c|}
\hline Variables & Response & Acceptance (rate, \%) & OR & $95 \% \mathrm{CI}$ & $P$ value \\
\hline \multirow{2}{*}{ Age (years) } & Younger $(<28)$ & $133(57.3)$ & 0.64 & $0.30-1.35$ & 0.243 \\
\hline & Older $(\geq 28)$ & $102(49.5)$ & 1 & & \\
\hline \multirow{2}{*}{ Education } & At least high school & $142(56.8)$ & 1.34 & $0.92-1.96$ & 0.128 \\
\hline & Less than high school & $93(49.5)$ & 1 & & \\
\hline \multirow{2}{*}{ Occupation } & Unemployed & $147(51.2)$ & 1.29 & $0.86-1.92$ & 0.219 \\
\hline & Employed & $85(56.3)$ & 1 & & \\
\hline \multirow{2}{*}{ Monthly income (RMB) } & $\geq 1000$ & $150(51.9)$ & 0.81 & $0.55-1.21$ & 0.307 \\
\hline & $<1000$ & $85(57.0)$ & 1 & & \\
\hline \multirow{3}{*}{ Looking for sexual partners place } & Internet & $103(50.2)$ & 0.76 & $0.47-1.25$ & 0.281 \\
\hline & Park & $77(57.9)$ & 1.04 & $0.61-1.77$ & 0.892 \\
\hline & Others & $53(53.0)$ & 1 & & \\
\hline \multirow{2}{*}{ AIDS prevention knowledge scores } & $10-11$ & $112(59.9)$ & 1.55 & $1.06-2.28$ & 0.024 \\
\hline & $<10$ & $123(49.0)$ & 1 & & \\
\hline \multirow{3}{*}{ Previous times of HIV testing within VCT } & $>3$ & $88(42.5)$ & 2.91 & $1.43-5.92$ & 0.003 \\
\hline & $1-3$ & $131(67.9)$ & 1.02 & $0.51-2.05$ & 0.963 \\
\hline & None tested before & $16(42.1)$ & 1 & & \\
\hline \multirow{2}{*}{ PITC benefited oneself } & Yes & $182(53.1)$ & 0.9 & $0.57-1.42$ & 0.638 \\
\hline & No & $53(55.8)$ & 1 & & \\
\hline \multirow{2}{*}{ PITC benefited families and friends } & Yes & $165(61.1)$ & 2.2 & $1.49-3.26$ & $<0.001$ \\
\hline & No & $70(41.7)$ & 1 & & \\
\hline \multirow{2}{*}{ Getting AIDS prevention knowledge from radio } & Yes & $138(64.8)$ & 2.43 & $1.65-3.57$ & $<0.001$ \\
\hline & No & $97(43.3)$ & 1 & & \\
\hline \multirow{2}{*}{ Getting AIDS prevention knowledge from TV } & Yes & $167(59.9)$ & 2 & $1.34-2.96$ & 0.001 \\
\hline & No & $68(43.0)$ & 1 & & \\
\hline \multirow{2}{*}{ Getting AIDS prevention knowledge from newspaper } & Yes & $170(61.6)$ & 2.39 & $1.61-3.56$ & $<0.001$ \\
\hline & No & $65(40.4)$ & 1 & & \\
\hline \multirow{2}{*}{ Getting AIDS prevention knowledge from friends } & Yes & $164(57.1)$ & 1.5 & $1.01-2.23$ & 0.044 \\
\hline & No & $71(47.3)$ & 1 & & \\
\hline \multirow{2}{*}{ Getting AIDS prevention knowledge from leaflet } & Yes & $167(62.8)$ & 2.58 & $1.74-3.83$ & $<0.001$ \\
\hline & No & $68(39.8)$ & 1 & & \\
\hline \multirow{2}{*}{ Getting AIDS prevention knowledge from Internet } & Yes & $171(58.8)$ & 1.85 & $1.24-2.76$ & 0.003 \\
\hline & No & $64(43.8)$ & 1 & & \\
\hline \multirow{2}{*}{ Self-identity high risk of HIV } & Yes & $36(72.0)$ & 2.44 & $1.28-4.67$ & 0.007 \\
\hline & No & $199(51.3)$ & 1 & & \\
\hline \multirow{2}{*}{ Self-identity high risk of STD } & Yes & $194(55.9)$ & 1.55 & $0.97-2.46$ & 0.066 \\
\hline & No & $41(45.1)$ & 1 & & \\
\hline
\end{tabular}

* PITC: provider-initiated HIV testing and counseling; VCT: HIV voluntary counseling and testing; OR: adjusted odds ratio.

from televised public advertisements; obtaining HIV/AIDS prevention knowledge from newspapers and magazines; obtaining HIV/AIDS prevention knowledge from friends; obtaining HIV/AIDS prevention knowledge from brochures; obtaining HIV/AIDS prevention knowledge from the Internet.

In the final adjusted multivariate model (Table 3), the strongest predictors of an MSM's acceptance of PITC were having been previously tested for HIV more than three times in a VCT clinic (OR $=2.95,95 \% \mathrm{CI}$ : $1.36-6.37, P=0.006)$, considering PITC beneficial to family and friends (OR = 1.91, 95\% CI: $1.25-2.92, P=0.003)$, having obtained AIDS prevention knowledge from brochures $(\mathrm{OR}=2.52,95 \% \mathrm{CI}$ :
1.64-3.87, $P<0.001)$, having obtained AIDS prevention knowledge from the Internet (OR $=1.66,95 \%$ CI: $1.07-2.58$, $P=0.025)$, and self-identification as being at high risk for HIV infection $(\mathrm{OR}=2.84,95 \% \mathrm{CI}: 1.37-5.91, P=0.005)$.

\section{Discussion}

This study was the first to explore the acceptance of PITC among Shenyang MSM. Understanding various characteristics of this population is important for drawing conclusions that have plausible implication for the prevention and control of China's nationwide HIV/AIDS epidemic. In the present study, the proportion of MSM who had not previously 
TABLE 3: Willingness for PITC. Multivariate analysis.

\begin{tabular}{|c|c|c|c|}
\hline Variables & Response & OR $(95 \% \mathrm{CI})$ & $P$ value \\
\hline \multirow{3}{*}{ Previous HIV testing times within VCT } & $>3$ & $2.95(1.36-6.37)$ & 0.006 \\
\hline & $1-3$ & $0.94(0.44-2.02)$ & 0.866 \\
\hline & None tested bef & & \\
\hline \multirow{2}{*}{ PITC brought benefit to family and friends } & Yes & $1.91(1.25-2.92)$ & 0.003 \\
\hline & No & & \\
\hline \multirow{2}{*}{ Gained AIDS prevention knowledge from brochures } & Yes & $2.52(1.64-3.87)$ & $<0.001$ \\
\hline & No & & \\
\hline \multirow{2}{*}{ Gained AIDS prevention knowledge from Internet } & Yes & $1.66(1.07-2.58)$ & 0.025 \\
\hline & No & & \\
\hline \multirow{2}{*}{ Self-identity high risk of HIV } & Yes & $2.84(1.37-5.91)$ & 0.005 \\
\hline & No & & \\
\hline
\end{tabular}

" PITC: provider-initiated HIV testing and counseling; VCT: HIV voluntary counseling and testing; OR: adjusted odds ratio.

heard of PITC was nearly $45 \%$. After learning about PITC, $53.7 \%(235 / 438)$ of participants considered it necessary to implement PITC in hospitals. This percentage is slightly lower compared to attitudes reported in another study conducted on Chinese female sex workers (FSWs) in which the median acceptance of PITC was 59.8\% [21]. These percentages suggest that the MSM subpopulation is just as important target as FSWs. Another study involving TB patients showed that their acceptance rate of PITC was $99.1 \%$ in Guangxi province, southern China [22]. Regarding resistance to PITC-for reasons that include the fear of receiving a positive test result or a self-evaluation of being at no or low risk for HIV infection-the proportion of MSM refusing PITC is higher than that of other high-risk populations [15]. Because Chinese society emphasizes the traditional obligation to uphold familial reputations and lineages, MSM are threatened by the stigma associated with the exposure of their sexual orientation in China $[5,23]$, resulting in a fear of seeking out HIV-related information, resources, and testing. Therefore, this study must be applied together with efforts to promote tolerance of sexual orientation and oppose discrimination. In summary, more health education and social intervention should target MSM, and more resources should be allocated to HIV prevention among MSM.

Participants who declined PITC gave reasons such as fear of having their privacy or rights violated and identifying themselves as being at no or low risk for HIV infection. In the United States, "having no risk for HIV infection" is also a common reason for declining routine HIV testing $[16,24]$. These findings confirm that perception of risk is one of the main reasons that participants declined testing for HIV. Our surveys demonstrated that people who self-identify as being at high risk for HIV infection were more likely to accept PITC (OR $=2.84,95 \%$ CI: $1.37-5.91)$. This information strongly suggests that patients need to be educated about the actual risks and the realities of HIV infection. We also found that those who learned about AIDS prevention from brochures were more willing to accept PITC ( $\mathrm{OR}=2.52,95 \%$ CI: 1.64-3.87). This finding implies that, when offering HIV testing, health care providers should provide educational printed materials about HIV risk factors. This information can help patients better understand their personal risks and may decrease the number of patients who decline testing based on their perception of their risk.

Also, our results highlight a visible relationship between being aware that HIV prevention is important and accepting PITC (OR $=0.024,95 \%$ CI: $1.06-2.28)$. Although the scores from the HIV prevention knowledge portion of the questionnaire were not entered into the multivariate analysis model of PITC acceptance factors, our study found that only $21.2 \%$ (93/438) of participants answered all 11 HIV prevention questions correctly. Meanwhile, the rates of condom use with regular and casual sexual partners were both low $24.7 \%$ with regular sexual partners and $28.7 \%$ with casual sexual partners). Similarly, MSM participants from Beijing and Jinan had lower awareness of AIDS knowledge and lower rates of condom use [25, 26]. AIDS prevention knowledge informs attitudes about AIDS and the development of safe sexual habits. Thus, to enhance basic HIV/AIDS knowledge among MSM in Shenyang, targeted health education should be carried out and sustained.

Furthermore, multivariate analysis found that MSM who had been tested for HIV at VCT clinics more than three times showed significantly higher acceptance of PITC compared to MSM who never received VCT (OR $=2.95,95 \%$ CI: $1.36-$ 6.37). This finding illustrates that VCT plays a critical role in MSM consultations, education, and intervention. Despite there being more than 4000 VCT sites in China in 2007, the average number of attendees was less than two per day [27, 28]. These numbers underscore the urgent need for greater efforts to increase VCT utilization in China. Only $19.1 \%$ of VCT clinics were located in hospitals in 2007 [27]. In 2009, $28.9 \%$ of hospitals reported having a VCT clinic according to data from Internet-based reporting of HIV/AIDS in China, compared with $25.0 \%$ in $2008[29,30]$. Patients who had been tested for HIV in hospitals were tested as part of the routine examination of inpatients. This phenomenon implies that expanding HIV testing to all outpatients would greatly increase the number of people being tested. Because of the double stigma attached to being HIV positive and homosexual, MSM may avoid testing and treatment facilities to avoid the potential for discrimination [31]. Interestingly, 
many participants preferred accepting HIV testing in hospitals rather than in testing and treatment facilities [8]. HIV/AIDS patients can receive integrated services, such as referrals, intervention, treatment, and timely care, if they are diagnosed with HIV in a hospital. Thus, the government should continue its support of VCT for AIDS prevention, work on increasing PITC acceptance among MSM, and design methodologies for earlier HIV detection among MSM to reduce the risk of secondary HIV transmission.

Another important finding was that the Internet was the most common source $(46.8 \%)$ for seeking male sex partners for the participants in our study. We also found that those learning about AIDS prevention from the Internet showed higher rates of PITC acceptance (OR $=1.66$, 95\% CI: $1.07-$ 2.58). Studies have indicated that MSM who often used the Internet have more favourable attitudes toward online methods of promoting the prevention of STIs (sexually transmitted infections)/HIV [32]. Zhang's study showed that more than one-third of respondents reported using the Internet to research health information [33]. These findings show that the Internet is an effective means of providing HIV prevention and health education information to MSM and, therefore, should be part of forefront intervention strategies for PITC education.

We also found that MSM who believed PITC could benefit their family and friends displayed higher rates of PITC acceptance $(\mathrm{OR}=1.91,95 \% \mathrm{CI}: 1.25-2.92)$. Due to social pressures and traditional family values, approximately $70-80 \%$ of Chinese MSM are currently, or will eventually get, married to a woman [34]. The influence of traditional Chinese culture causes Chinese people to see their families as their main source of support in cases of severe disease. This impact of culture on behaviour demonstrates that widespread family-based HIV prevention strategies that utilize health education should be encouraged.

We recognize several limitations of our study. First, selection bias may have influenced the results of this study because the participants were a convenient sample of MSM who live in Shenyang. Results from this study may not be applicable to the greater MSM population of China. Secondly, the survey contained questions about sensitive topics, and participants may have felt uncomfortable answering them completely honestly. To minimize such bias, we asked volunteers from local NGOs to act as the interviewers for the study and provided special training for the purposes of this study. Finally, placing the survey location in a hospital might have encouraged PITC acceptance among our study population, creating a social desirability bias.

Despite these limitations, our study provides valuable insight and lessons regarding PITC acceptance among Chinese high-risk MSM. Our results suggest that strengthening health education on the Internet and improving the utilization of VCTs may increase the willingness to be tested for HIV among Chinese MSM.

\section{Conclusions}

Our study found that $53.7 \%$ of the participants reported a willingness to accept PITC. Additionally, MSM who received
VCT more than three times, obtained HIV/AIDS knowledge from brochures or the Internet, and were highly aware of their own HIV risk were most willing to accept PITC. Our results indicate that much greater efforts are needed to lower the barriers to PITC acceptance, improve HIV/AIDS and PITC knowledge, and promote the extension of HIV testing in order to, consequently, improve PITC acceptance among MSM in China, particularly among groups engaged in risky behavior and have a high HIV occurrence.

\section{Acknowledgments}

The authors thank the staff at Shenyang Sunny Workgroup for their help with MSM participant enrollment and the staff at the HIV Voluntary Counseling and Testing Clinic at the First Affiliated Hospital of China Medical University for their generous help in project implementation. The authors also thank Christina Liao from the North American entering class of 2012 at China Medical University and Jason Tung, who helped review this paper. Finally, the authors thank all who participated in the study. This study was funded by the Mega-Projects of National Science Research for the 12th Five-Year Plan (2012ZX10001006); China-Gates Foundation Cooperation Programme (2012)13; National Nature Science Foundation of China (81001291).

\section{References}

[1] T. J. Coates, O. A. Grinstead, S. E. Gregorich et al., "Efficacy of voluntary HIV-1 counselling and testing in individuals and couples in Kenya, Tanzania, and Trinidad: a randomised trial," The Lancet, vol. 356, no. 9224, pp. 103-112, 2000.

[2] J. A. Denison, K. R. O’Reilly, G. P. Schmid, C. E. Kennedy, and M. D. Sweat, "HIV voluntary counseling and testing and behavioral risk reduction in developing countries: a metaanalysis, 1990-2005," AIDS and Behavior, vol. 12, no. 3, pp. 363373, 2008.

[3] C. G. Beckwith, T. P. Flanigan, C. Del Rio et al., "It is time to implement routine, not risk-based, HIV testing," Clinical Infectious Diseases, vol. 40, no. 7, pp. 1037-1040, 2005.

[4] P.R.China Ministry of Health, UNAIDS, World Health Organization. A Joint Assessment of HIV/AIDS Prevention Treatment and Care in China, 2011, http://www.moh.gov.cn/.

[5] H. Shang, J. J. Xu, X. X. Han et al., "HIV prevention: bring safe sex to China," Nature, vol. 485, no. 7400, pp. 576-577, 2012.

[6] D. Holtgrave and J. McGuire, "Impact of counseling in voluntary counseling and testing programs for persons at risk for or living with HIV infection," Clinical Infectious Diseases, vol. 45, supplement 4, pp. S240-S243, 2007.

[7] M. Sweat, S. Gregorich, G. Sangiwa et al., "Cost-effectiveness of voluntary HIV-1 counselling and testing in reducing sexual transmission of HIV-1 in Kenya and Tanzania," The Lancet, vol. 356, no. 9224, pp. 113-121, 2000.

[8] T. Zhang, J. Zhang, M. Gao, N. He, and R. Detels, "Knowledge, attitudes and practices of voluntary HIV counselling and testing among rural migrants in central China: a cross-sectional study," European Journal of Public Health, vol. 22, no. 2, pp. 192-197, 2012.

[9] T. Cannell Nieburg P and J. S. Morrison, Expanded HIV Testing: Critical Gateway to HIV Treatment and Prevention Requires 
Major Resources, Effective Protections, Center for Strategic and International Studies, Washington, DC, USA, 2005, http://csis .org/files/media/csis/pubs/0501_expandedhivtesting.pdf.

[10] WHO, Guidance on Provider-Initiated HIV Testing and Counseling in Health Facilities, World Health Organization, Geneva, Switzerland, 2007.

[11] B. M. Branson, H. H. Handsfield, M. A. Lampe et al., "Revised recommendations for HIV testing of adults, adolescents, and pregnant women in health-care settings," MMWR Recommendations and Reports, vol. 55, no. -14, pp. 1-17, 2006.

[12] I. V. Bassett and R. P. Walensky, "Integrating HIV screening into routine health care in resource-limited settings," Clinical Infectious Diseases, vol. 50, supplement 3, pp. S77-S84, 2010.

[13] L. C. Ivers, K. A. Freedberg, and J. S. Mukherjee, "Providerinitiated HIV testing in rural Haiti: low rate of missed opportunities for diagnosis of HIV in a primary care clinic," AIDS Research and Therapy, vol. 4, article 28, 2007.

[14] J. Pattanaphesaj and Y. Teerawattananon, "Reviewing the evidence on effectiveness and cost-effectiveness of HIV prevention strategies in Thailand," BMC Public Health, vol. 10, article 401, 2010.

[15] R. L. J. Heijman, I. G. Stolte, H. F. J. Thiesbrummel et al., “Opting out increases HIV testing in a large sexually transmitted infections outpatient clinic," Sexually Transmitted Infections, vol. 85, no. 4, pp. 249-255, 2009.

[16] J. Brown, I. Kuo, J. Bellows et al., "Patient perceptions and acceptance of routine emergency department HIV testing," Public Health Reports, vol. 123, supplement 3, pp. 21-26, 2008.

[17] D. Nakanjako, M. Kamya, K. Daniel et al., "Acceptance of routine testing for HIV among adult patients at the medical emergency unit at a national referral hospital in Kampala, Uganda," AIDS and Behavior, vol. 11, no. 5, pp. 753-758, 2007.

[18] D. H. Liang and J. Li, "The preliminary study of civil nonprofit organizations in the programe of MSM health education," Chinese Journal of AIDS \& STD, vol. 12, pp. 271-284, 2006.

[19] J.-J. Xu, M. Zhang, K. Brown et al., "Syphilis and HIV seroconversion among a 12-month prospective cohort of men who have sex with men in Shenyang, China," Sexually Transmitted Diseases, vol. 37, no. 7, pp. 432-439, 2010.

[20] Z. X. Chu, N. Ma, J. J. Xu et al., "HIV prevalence and its associated factors among 2074 men who have sex with men(MSM) in Liaoning province, China," Chinese Journal of Public Health, vol. 27, pp. 967-969, 2011.

[21] X. Jin, N. Wang, and G.-W. Ding, "Correlates of and willingness to participate in HIV routine counseling and testing among female sex workers in a city of Yunnan province," Chinese Journal of Epidemiology, vol. 30, no. 2, pp. 205-206, 2009.

[22] X.-W. Wang, Y. Liu, B.-Q. Dong, F.-Y. Liu, and Q. Chen, "Provider-initiated testing and counselling for human immunodeficiency virus among tuberculosis patients in Guangxi," International Journal of Tuberculosis and Lung Disease, vol. 14, no. 7, pp. 921-923, 2010.

[23] K. Yun, J. J. Xu, K. H. Reilly et al., "Prevalence of bisexual behaviour among bridge population of men who have sex with men in China: a meta-analysis of observational studies," Sexually Transmitted Infections, vol. 87, no. 7, pp. 563-570, 2011.

[24] R. V. Liddicoat, E. Losina, M. Kang, K. A. Freedberg, and R. P. Walensky, "Refusing HIV testing in an urgent care setting: results from the "think HIV" program," AIDS Patient Care and STDs, vol. 20, no. 2, pp. 84-92, 2006.
[25] H. Liu, Y. Liu, Y. Xiao et al., "A survey of the knowledge, attitude, andbehaviors on STD/AIDS in men who have sex with men in Beijing," Chinese Journal of AIDS \& STD, vol. 11, no. 4, pp. 268270, 2005.

[26] Y. W. Zhu, S. M. Ruan, H. Yang et al., "Investigation on AIDS related knowledge, risk behavior and HIV infection in MSM in Jinan city," Preventive Medicine Tribune, vol. 13, no. 6, pp. 490492, 2007.

[27] State Council AIDS Working Committee Office Ministry of Health of China, UN Theme Group on HIV/AIDS in China, A Joint Assessment of HIV/AIDS Prevention, Treatment and Care in China, 2007, http://www.chinaids.org.cn.

[28] Y. Wang, B. Li, J. Zheng et al., "Factors related to female sex workers' Willingness to utilize VCT service: a qualitative study in Jinan City, Northern China," AIDS and Behavior, vol. 13, no. 5, pp. 866-872, 2009.

[29] Q. Q. Qin, L. Wang, Z. W. Ding et al., "Quality analysis of national HIV/AIDS online case reporting in 2008," Practical Preventive Medicine, vol. 25, pp. 1785-1787, 2009.

[30] L. N. Sun, Q. Q. Qin, Z. W. Ding, and L. Wang, "Performance of internet-based reporting of HIV /AIDS in China, 2009," Disease Surveillance, vol. 25, no. 11, pp. 907-911, 2010.

[31] Z. Wu, X. Sun, S. G. Sullivan, and R. Detels, "HIV testing in China," Science, vol. 312, no. 5779, pp. 1475-1476, 2006.

[32] G. Bolding, M. Davis, L. Sherr, G. Hart, and J. Elford, "Use of gay Internet sites and views about online health promotion among men who have sex with men," AIDS Care, vol. 16, no. 8, pp. 9931001, 2004.

[33] D. Zhang, P. Bi, F. Lv, H. Tang, J. Zhang, and J. E. Hiller, "Internet use and risk behaviours: an online survey of visitors to three gay websites in China," Sexually Transmitted Infections, vol. 83, no. 7, pp. 571-576, 2007.

[34] B. C. Zhang, X. F. Li, Q. S. Chu et al., "A survey of HIV/AIDS related behaviors among $2250 \mathrm{MSM}$ in nine major cities of China," Chinese Journal of AIDS \& STD, vol. 14, no. 6, pp. 451457, 2008. 


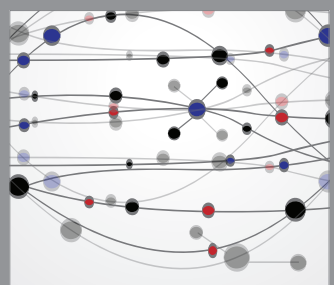

The Scientific World Journal
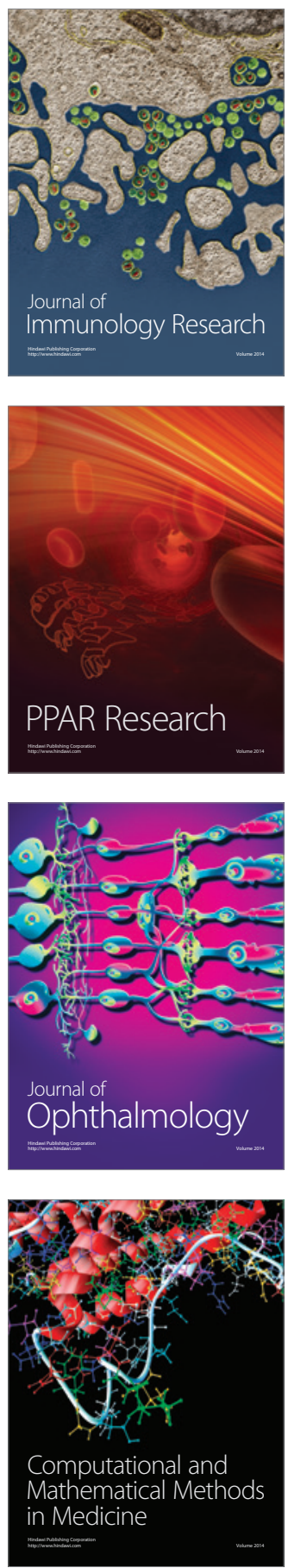

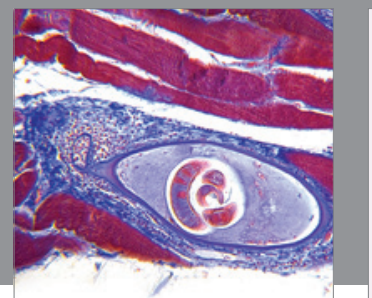

Gastroenterology

Research and Practice
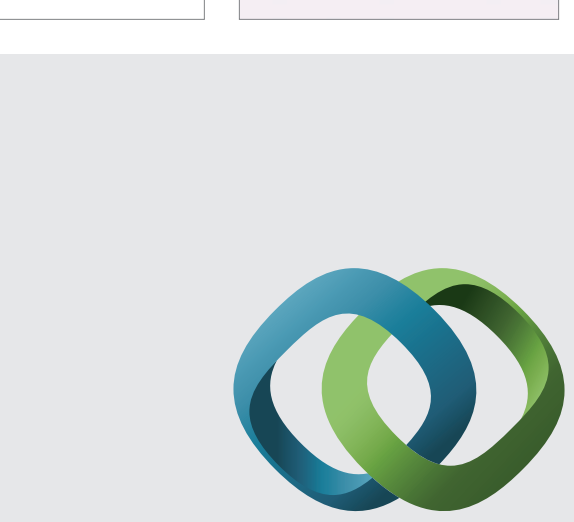

\section{Hindawi}

Submit your manuscripts at

http://www.hindawi.com
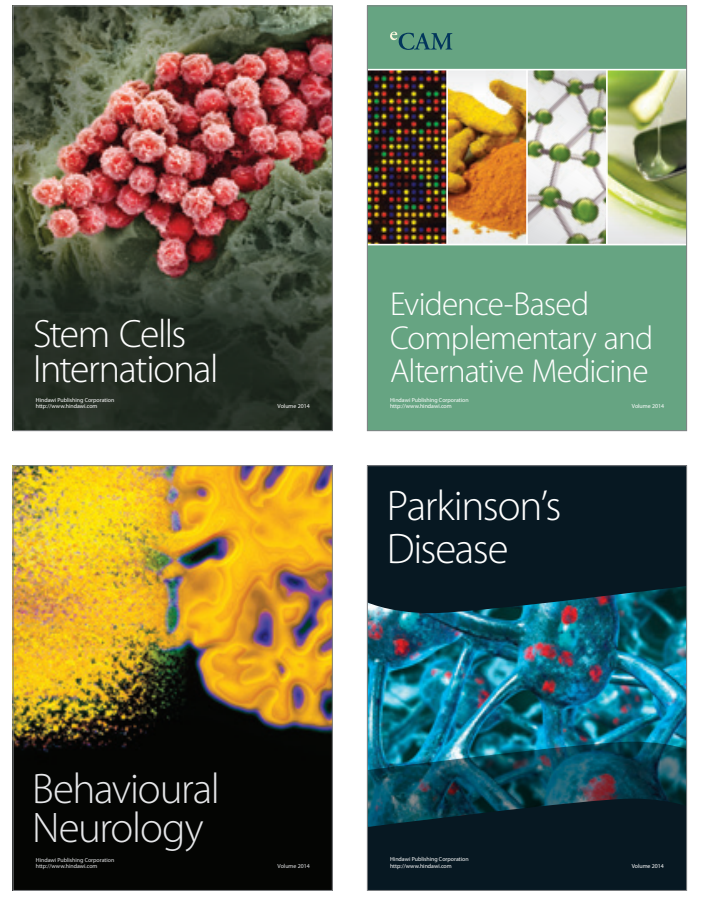
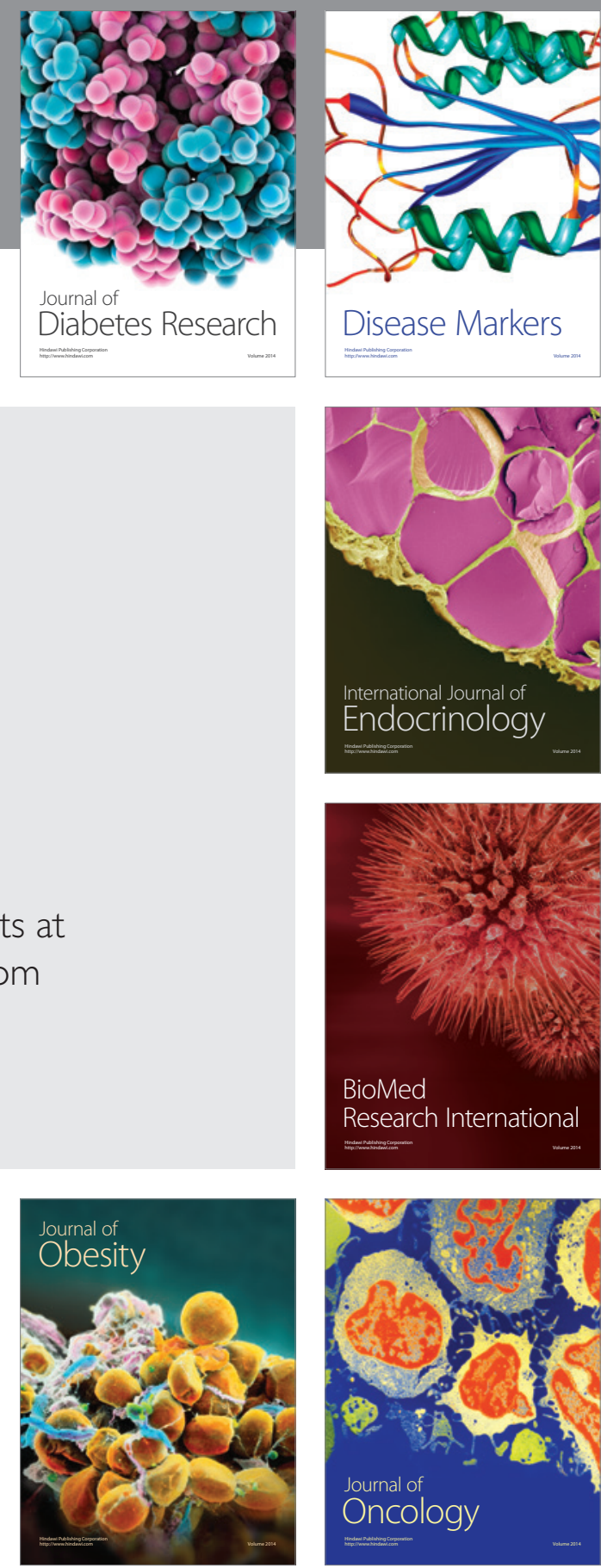

Disease Markers
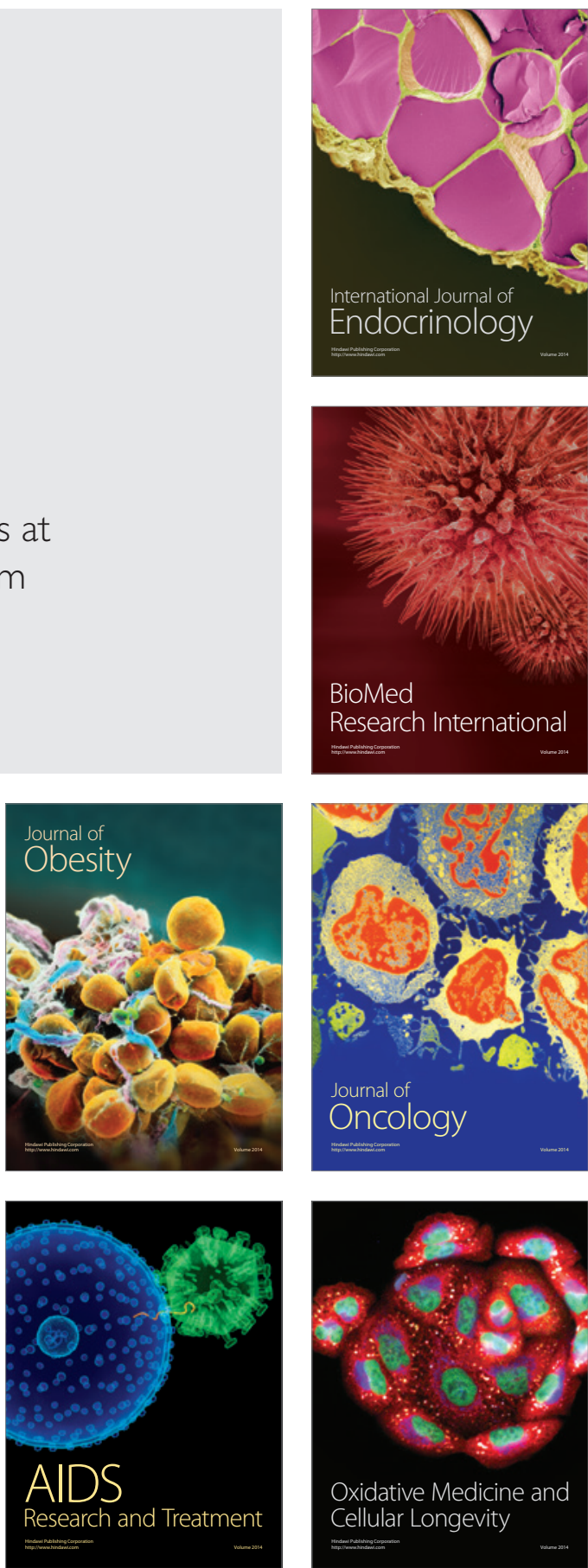